\title{
The essential spectrum of holomorphic Toeplitz operators on $H^{p}$ spaces
}

\author{
by \\ Mats Andersson and Sebastian Sandberg (Göteborg)
}

Abstract. We compute the essential Taylor spectrum of a tuple of analytic Toeplitz operators $T_{g}$ on $H^{p}(D)$, where $D$ is a strictly pseudoconvex domain. We also provide specific formulas for the index of $T_{g}$ provided that $g^{-1}(0)$ is a compact subset of $D$.

1. Introduction and main results. Let $D$ be a bounded domain in $\mathbb{C}^{n}$ with $C^{3}$ boundary. The Hardy space $H^{p}$ consists of the holomorphic functions $f$ in $D$ such that

$$
\|f\|_{H^{p}}^{p}=\limsup _{\varepsilon \searrow 0} \int_{\partial D_{\varepsilon}}|f|^{p} d \sigma<\infty
$$

where $D_{\varepsilon}=\{z \in D ; \varrho(z)<-\varepsilon\}$ for some defining function $\varrho$ of $D$, and $H^{\infty}$ denotes the space of bounded holomorphic functions. A tuple $g_{1}, \ldots, g_{m}$ of bounded holomorphic functions on $D$ defines a tuple $T_{g}$ of commuting Toeplitz operators on $H^{p}(D)$, i.e., $T_{g_{j}} f=g_{j} f$. For each fixed $w \in \mathbb{C}^{m}$ we have the Koszul complex

$$
0 \leftarrow \Lambda^{0} H^{p} \stackrel{\delta_{w-g}}{\longleftarrow} \Lambda^{1} H^{p} \stackrel{\delta_{w-g}}{\longleftarrow} \ldots \stackrel{\delta_{w-g}}{\longleftarrow} \Lambda^{m} H^{p} \leftarrow 0,
$$

where $\Lambda^{l} H^{p}$ is the space of all formal expressions

$$
f=\sum_{|I|=l}^{\prime} f_{I} e_{I},
$$

$e_{I}=e_{I_{1}} \wedge \ldots \wedge e_{I_{l}}, e_{1}, \ldots, e_{m}$ being some abstract basis, the prime means that the summation is performed over increasing multiindices $I$ of length $l$, and the mappings $\delta_{w-g}$ are contraction (interior multiplication) with the

2000 Mathematics Subject Classification: 32A26, 32A35, 47A10, 47A13.

Key words and phrases: Toeplitz operator, $H^{p}$ corona problem, Koszul complex, $\bar{\partial}$-complex, strictly pseudoconvex domain, Hardy space, Taylor spectrum, essential Taylor spectrum.

Research of M. Andersson partially supported by the Swedish Natural Science Research Council. 
operator-valued dual object

$$
\sum_{j=1}^{m}\left(w_{j}-g_{j}\right) e_{j}^{*},
$$

where $e_{j}^{*}$ is the dual basis. If $\Lambda^{1} H^{p}$ is identified with $\left(H^{p}\right)^{m}$, then the next to leftmost arrow in (1.1) is just the mapping $\left(H^{p}\right)^{m} \rightarrow H^{p}$ defined by

$$
\left(u_{1}, \ldots, u_{m}\right) \mapsto \sum\left(w_{j}-g_{j}\right) u_{j} .
$$

The Taylor spectrum $\sigma\left(T_{g}, H^{p}\right)$ of the (commuting tuple of operators) $T_{g}$ on $H^{p}$ is defined as the set of $w \in \mathbb{C}^{m}$ such that (1.1) is not exact. The right spectrum $\sigma_{\mathrm{r}}\left(g, H^{p}\right)$ is the set of $w$ such that (1.1) is not exact at the next to leftmost point, i.e., such that (1.2) is not surjective. It is a consequence of the open mapping theorem (see [11]) that $\sigma_{\mathrm{r}}\left(g, H^{p}\right)$ and $\sigma\left(g, H^{p}\right)$ are closed and hence compact sets. Obviously (1.2) is not surjective if $w \in g(D)$, and therefore we have the inclusions

$$
\overline{g(D)} \subset \sigma_{\mathrm{r}}\left(g, H^{p}\right) \subset \sigma\left(g, H^{p}\right) .
$$

The essential spectrum $\sigma_{\text {ess }}\left(T_{g}, H^{p}\right)$ is the set of $w \in \mathbb{C}^{m}$ such that not all the homology of (1.1) is finite-dimensional, or stated in other words, that $T_{w-g}$ is not a Fredholm tuple. Moreover, the right essential spectrum $\sigma_{\text {ress }}\left(T_{g}\right)$ is the set of $w$ such that the homology of (1.1) at $l=0$ is infinite-dimensional. In [3] the following was proved:

Theorem 1.1. Let $D$ be a strictly pseudoconvex domain in $\mathbb{C}^{n}$ with $C^{3}$ boundary and assume that $g_{1}, \ldots, g_{m} \in H^{\infty}$. Then $\sigma\left(g, H^{p}\right)=\sigma_{\mathrm{r}}\left(g, H^{p}\right)=$ $\overline{g(D)}$ for all $p<\infty$. If $n=1$ this also holds for $p=\infty$.

The main result in this note is

TheOREm 1.2. Let $D$ be a strictly pseudoconvex domain in $\mathbb{C}^{n}$ with $C^{3}$ boundary and assume that $g_{1}, \ldots, g_{m} \in H^{\infty}$. Then

$$
\sigma_{\text {ess }}\left(T_{g}, H^{p}\right)=\sigma_{\text {ress }}\left(T_{g}, H^{p}\right)=\bigcap_{\text {open } \mathcal{U} \supset \partial D} \overline{g(D \cap \mathcal{U})}
$$

for all $p<\infty$. If $n=1$ this also holds for $p=\infty$.

Thus, roughly speaking, $\sigma_{\text {ess }}\left(T_{g}\right)$ is the image of the boundary of $\partial D$ under the mapping $g: D \rightarrow \mathbb{C}^{m}$. It follows that $\sigma_{\text {ess }}\left(T_{g}\right)=\sigma\left(T_{g}\right)$ if $m<n$. For two generators these results were proved in [9]. The analog of Theorem 1.1 for the Bergman space $L_{\mathrm{a}}^{2}(D)=\mathcal{O}(D) \cap L^{2}(D)$ was proved in [5] for any bounded pseudoconvex domain, together with the inclusion

$$
\sigma_{\text {ess }}\left(T_{g}, L_{\mathrm{a}}^{2}(D)\right) \subset \bigcap_{\text {open } \mathcal{U} \supset \partial D} \overline{g(D \cap \mathcal{U})},
$$

with equality at least if $D$ is strictly pseudoconvex. 
REMARK 1. Theorem 1.1 was also proved for BMOA and $\frac{L_{\mathrm{a}}^{p}(D)}{g(D)}=$ $\mathcal{O}(D) \cap L^{p}(D), 1 \leq p<\infty$, in [3]. The equality $\sigma_{\mathrm{r}}\left(T_{g}, X\right)=\overline{g(D)}$ has been proved for a large number of other function spaces $X$ in strictly pseudoconvex domains such as various Besov spaces ([7] and [8]) and $\mathcal{Q}_{p}$ spaces ([13] and [4]). In each case, the $g_{j}$ are assumed to be multipliers on the space $X$ in question. The proofs in these papers can be adapted to yield the complete analogues of Theorem 1.1 and the corresponding inclusions (1.5). Moreover, Theorem 1.1 holds for all $p \leq 2$ if $D$ admits a $C^{2}$ plurisubharmonic defining function (see [12], [1], and [2]), and again one can prove the corresponding inclusion (1.5) in this case.

2. Proofs. The inclusion

$$
\bigcap_{\text {open } \mathcal{U} \supset \partial D} \overline{g(D \cap \mathcal{U})} \subset \sigma_{\text {ress }}\left(T_{g}, H^{p}\right)
$$

follows just as for the Bergman space $L_{\mathrm{a}}^{2}(D)$ (see Theorem 8.2.6 in [6]). Since clearly $\sigma_{\text {ress }}\left(T_{g}, H^{p}\right) \subset \sigma_{\text {ess }}\left(T_{g}, H^{p}\right)$, we will focus on the remaining inclusion

$$
\sigma_{\text {ess }}\left(T_{g}, H^{p}\right) \subset \bigcap_{\text {open } \mathcal{U} \supset \partial D} \overline{g(D \cap \mathcal{U})} .
$$

for the rest of this paper. A moment's thought reveals that it is equivalent to the statement that (1.1) has finite-dimensional homology for each $w$ such that $g^{-1}(w)$ is a compact subset of $D$. In what follows we can with no loss of generality assume that $w=0$. Let us introduce the notation

$$
H_{r}\left(T_{g}, H^{p}(D)\right)=\frac{\operatorname{Ker}\left(\Lambda^{r} H^{p}(D) \stackrel{\delta_{g}}{\rightarrow} \Lambda^{r-1} H^{p}(D)\right)}{\operatorname{Im}\left(\Lambda^{r+1} H^{p}(D) \stackrel{\delta_{g}}{\rightarrow} \Lambda^{r} H^{p}(D)\right)}
$$

and define $H_{r}\left(T_{g}, \mathcal{O}(V)\right)$ in the analogous way, for $V \subset \subset D$. The restriction mapping $H^{p}(D) \rightarrow \mathcal{O}(V)$ then induces a mapping of complexes from (1.1) (for $w=0$ ) to the complex

$$
0 \leftarrow \Lambda^{0} \mathcal{O}(V) \stackrel{\delta_{g}}{\longleftarrow} \Lambda^{1} \mathcal{O}(V) \leftarrow \ldots,
$$

and hence we get a mapping on homology

$$
H_{r}\left(T_{g}, H^{p}(D)\right) \rightarrow H_{r}\left(T_{g}, \mathcal{O}(V)\right)
$$

We will prove the following theorem.

THEOREM 2.1. Suppose that $D$ is a strictly pseudoconvex domain with $C^{3}$ boundary, and $g$ is a tuple of bounded holomorphic functions on $D$ such that $g^{-1}(0)$ is a compact subset of $D$, and let $V$ be a pseudoconvex open set such that $g^{-1}(0) \subset V \subset \subset D$. If $n \geq 1$ and $1 \leq p<\infty$ or $n=1$ and $p=\infty$, then the mapping (2.2) is an isomorphism. 
If $g^{-1}(0)$ is a compact subset of $D$ it is clearly a discrete set. It is well known that then the spaces $H_{r}\left(T_{g}, \mathcal{O}(V)\right)$ are finite-dimensional. Taking Theorem 2.1 for granted we can therefore conclude that all $H_{r}\left(T_{g}, H^{p}(D)\right)$ are finite-dimensional as well. By the remark above this means that the inclusion (2.1) holds. Thus Theorem 1.2 is proved.

If $T_{g}$ is a Fredholm tuple we define the index as

$$
\operatorname{Ind}\left(T_{g}, H^{p}(D)\right)=\sum_{r=0}^{m}(-1)^{r} \operatorname{dim} H_{r}\left(T_{g}, H^{p}(D)\right),
$$

and $\operatorname{Ind}\left(T_{g}, \mathcal{O}(V)\right)$ in the analogous way. Then we have

COROLlary 2.2. Under the assumptions of Theorem 2.1 we have

$$
\operatorname{Ind}\left(T_{g}, H^{p}(D)\right)=\operatorname{Ind}\left(T_{g}, \mathcal{O}(V)\right) .
$$

In particular, if $m=n$ it is well known that $H_{r}\left(T_{g}, \mathcal{O}(V)\right)=0$ for $r>0$ and that

$$
\operatorname{dim} H_{0}\left(T_{g}, \mathcal{O}(V)\right)=\sum_{j=1}^{M} \nu_{z_{j}}(g)
$$

if $z_{1}, \ldots, z_{M}$ are the zeros of $g$ and $\nu_{z_{j}}(g)$ is the order of the zero of $g$ at the point $z_{j}$. Thus we have

COROLlary 2.3. Under the assumptions of Theorem 2.1 and $m=n$ we have $H_{r}\left(T_{g}, H^{p}(D)\right)=0$ for $r>0$ and

$$
\operatorname{Ind}\left(T_{g}, H^{p}(D)\right)=\sum_{j=1}^{M} \nu_{z_{j}}(g) .
$$

The formula (2.3) appeared in [5] for the Bergman space $L_{\mathrm{a}}^{2}(D)$ instead of $H^{p}(D)$.

3. Proof of Theorem 2.1. We only write down the proof of Theorem 2.1 for $H^{p}(D), 1 \leq p<\infty$; the case $p=\infty$ when $n=1$ follows with minor modifications. The proof relies on the technique and results of [10]. Fix a $p, 1 \leq p<\infty$, and let $X=H^{p}(D)$. Moreover, let $\mathcal{E}_{0, k}(D)$ denote the space of smooth $(0, k)$-forms in $D$, and $\mathcal{E}_{0, k}(\bar{D})$ the subspace of forms that are smooth up to the boundary. There are (cf. [10]) Fréchet spaces $B_{k}$ such that $\mathcal{E}_{0, k}(\bar{D}) \subset B_{k} \subset \mathcal{E}_{0, k}(D)$,

$$
0 \rightarrow X \rightarrow B_{0} \stackrel{\bar{\partial}}{\rightarrow} B_{1} \stackrel{\bar{\partial}}{\rightarrow} \ldots \stackrel{\bar{\partial}}{\rightarrow} B_{n} \rightarrow 0
$$

is a complex, the $T_{g_{j}}$ are bounded on $B_{k}$, and if $\chi$ is a cutoff function in $D$ which is identically 1 in a neighborhood of $g^{-1}(0)$, then also $(1-\chi) \bar{g}_{j} /|g|^{2}$ are bounded operators on $B_{k}$. In fact, we just take the intersections of the Banach spaces $B_{k}$ from [10] with $\mathcal{E}_{0, k}(D)$; the last statement follows from [10] 
since the $B_{k}$ condition is just a boundedness condition close to $\partial D$. To see that these new spaces are complete, let $\left\{f_{j}\right\}$ be a Cauchy sequence in $B_{k} \cap \mathcal{E}_{0, k}(D)$. Let $f$ be the limit of $\left\{f_{j}\right\}$ in $\mathcal{E}_{0, k}(D)$. Fatou's lemma applied twice to each term in the definition of $\|\cdot\|_{B_{k}}$ shows that $\left\|f-f_{j}\right\|_{B_{k}} \leq$ $\liminf _{l}\left\|f_{l}-f_{j}\right\|_{B_{k}}$. Thus $\left\{f_{j}\right\}$ converges to $f$ in $B_{k}$ and hence $B_{k} \cap \mathcal{E}_{0, k}(D)$ is complete.

Let $B_{k}^{l}(D)$ denote the space of $(0, k)$-forms $f$ that can be written as

$$
f=\sum_{|I|=l}^{\prime} f_{I} \wedge e_{I}
$$

where $f_{I} \in B_{k}$. We are taking the exterior algebra of both (the space generated by) $e_{1}, \ldots, e_{m}$ and $T_{0,1}^{*}(D)$, and therefore the operators $\delta=\delta_{g}$ and $\bar{\partial}$ anticommute so $B_{k}^{l}(D)$ is a double complex. We have the corresponding total complex

$$
\ldots \stackrel{\delta-\bar{\partial}}{\longrightarrow} \mathcal{L}^{r}\left(\operatorname{Tot} B_{k}^{l}(D)\right) \stackrel{\delta-\bar{\partial}}{\longrightarrow} \mathcal{L}^{r+1}\left(\operatorname{Tot} B_{k}^{l}(D)\right) \stackrel{\delta-\bar{\partial}}{\longrightarrow} \ldots
$$

where

$$
\mathcal{L}^{r}\left(\operatorname{Tot} B_{k}^{l}(D)\right)=\bigoplus_{p=0}^{n} B_{p}^{p-r}(D),
$$

and we let $H^{r}\left(\operatorname{Tot} B_{k}^{l}(D)\right)$ denote the cohomology of the complex (3.2). In the same way we have the double complex $\mathcal{E}_{0, k}^{l}(V)$, and its corresponding total complex $\mathcal{L}^{r}\left(\operatorname{Tot} \mathcal{E}_{0, k}^{l}(V)\right)$, and the cohomology groups $H^{r}\left(\operatorname{Tot} \mathcal{E}_{0, k}^{l}(V)\right)$. The restriction mapping induces a mapping of the double complexes, thus a mapping of the total complexes, and hence a mapping on cohomology, i.e., we have natural mappings

$$
H^{r}\left(\operatorname{Tot} B_{k}^{l}(D)\right) \rightarrow H^{r}\left(\operatorname{Tot} \mathcal{E}_{0, k}^{l}(V)\right) .
$$

Lemma 3.1. If $g^{-1}(0) \subset V \subset \subset D$, then (3.3) is an isomorphism.

Proof. Both $B_{k}^{l}(D)$ and $\mathcal{E}_{0, k}^{l}(V)$ are modules over $\mathcal{E}(\bar{D})$. Let

$$
\gamma=\sum_{j=1}^{m} \frac{\bar{g}_{j}}{|g|^{2}} e_{j}
$$

and let $\Gamma f=\gamma \wedge f$. Then the operator $\Gamma$ is bounded on $B_{k}\left(D \backslash g^{-1}(0)\right)$ and on $\mathcal{E}_{0, k}^{l}\left(V \backslash g^{-1}(0)\right)$, and $(\delta \Gamma+\Gamma \delta) f=f$ so the double complexes $B_{k}^{l}\left(D \backslash g^{-1}(0)\right)$ and $\mathcal{E}_{0, k}^{l}\left(V \backslash g^{-1}(0)\right)$ are exact in $l$ and therefore the lemma follows from a standard homology argument.

However, for us it is worthwhile to consider a more concrete version of the argument. Let $\chi$ be a cutoff function in $V$ that is 1 in a neighborhood 
of $g^{-1}(0)$; then $f \mapsto(1-\chi) \gamma \wedge f$ is a bounded operator on $B_{k}$. Moreover, if

$$
\alpha=\sum_{j=1}^{m} \gamma \wedge(\bar{\partial} \gamma)^{j-1},
$$

then $(\delta-\bar{\partial}) \alpha=1$ and $f \mapsto(1-\chi) \alpha \wedge f$ is bounded $\mathcal{L}^{r}\left(\operatorname{Tot} B_{k}^{l}(D)\right)$ $\rightarrow \mathcal{L}^{r-1}\left(\operatorname{Tot} B_{k}^{l}(D)\right)$. If now $[f] \in H^{r}\left(\operatorname{Tot} B_{k}^{l}(D)\right)$ and there is a $v \in$ $\mathcal{L}^{r}\left(\operatorname{Tot} \mathcal{E}_{0, k}^{l}(V)\right)$ such that $(\delta-\bar{\partial}) v=f$ in $V$, then

$$
(\delta-\bar{\partial})(\chi v+(1-\chi) \alpha \wedge f-\bar{\partial} \chi \wedge \alpha \wedge v)=f
$$

in $D$, which shows that (3.3) is injective. On the other hand, if $v \in$ $\mathcal{L}^{r}\left(\operatorname{Tot} \mathcal{E}_{0, k}^{l}(V)\right)$ and $(\delta-\bar{\partial}) v=0$, then

$$
(\delta-\bar{\partial})((1-\chi) \alpha \wedge v)=v-(\chi v-\bar{\partial} \chi \wedge \alpha \wedge v)
$$

which shows that $v$ is then cohomologous in $V$ to the form $\chi v-\bar{\partial} \chi \wedge \alpha \wedge v \in$ $\mathcal{L}^{r}\left(\right.$ Tot $\left.B_{k}^{l}(D)\right)$, proving that $(3.3)$ is surjective.

Notice that $\mathcal{E}_{0, k}^{l}(V)$ is exact in $k$ (recall that $V$ is assumed to be pseudoconvex) except on level $k=0$, where the kernels are $\mathcal{O}^{l}(V)=\Lambda^{l} \mathcal{O}(V)$. By standard homological algebra it follows that the natural mapping $H_{r}\left(T_{g}, \mathcal{O}(V)\right) \rightarrow H^{-r}\left(\operatorname{Tot} \mathcal{E}_{0, k}^{l}(V)\right)$ is an isomorphism (an explicit argument is contained in the proofs of Lemma 3.2 and Theorem 2.1 below). The mapping $X \rightarrow B_{0}$ also induces natural mappings $H_{r}\left(T_{g}, X\right) \rightarrow H^{-r}\left(\operatorname{Tot} B_{k}^{l}(D)\right)$ and thus we have the following picture:

$$
\begin{aligned}
H_{r}\left(T_{g}, X\right) \rightarrow H^{-r}\left(\operatorname{Tot} B_{k}^{l}(D)\right) & \simeq H^{-r}\left(\operatorname{Tot} \mathcal{E}_{0, k}^{l}(V)\right) \\
& \simeq H_{r}\left(T_{g}, \mathcal{O}(V)\right),
\end{aligned}
$$

where the composite mappings $H_{r}\left(T_{g}, X\right) \rightarrow H_{r}\left(T_{g}, \mathcal{O}(V)\right)$ are the natural ones, i.e., (3.3). If also the complex (3.1) were exact, then it would follow immediately, by the same argument, that the leftmost arrow in (3.4) were an isomorphism, and hence Theorem 2.1 would have been proved.

Unfortunately, we cannot find such a complex with the stated properties which is also exact. The main point in [10] is that one can do without exactness.

REMARK 2. This idea was used, though not formalized, in [3] and goes back to Wolff's proof of the corona problem. To see this, let $n=1$ and $p=\infty$. Then our space $B_{1}$ is the space of $(0,1)$-forms $f$ such that $r|f|^{2}+$ $r|\partial f|$ is a Carleson measure, $r$ being the distance to the boundary, and $B_{0}$ is the space of functions $u$ such that $\bar{\partial} u \in B_{1}$. Then $0 \rightarrow H^{\infty} \rightarrow B_{0} \rightarrow$ $B_{1} \rightarrow 0$ is a complex and $B_{j}$ are closed under $T_{g}$. Although not exact, any $f \in B_{1}$ admits a solution $u \in L^{\infty}(\partial D)$ to $\bar{\partial}_{b} u=f$ (this is Wolff's theorem), and this turns out to imply the missing isomorphism in (3.4) (for $r=0$ ). 
Assuming that $g^{-1}(0)$ is empty, as in the corona theorem, one concludes that $H_{r}\left(T_{g}, X\right)=0$, which is precisely the corona theorem.

It turns out that it is enough to find spaces $B_{k}^{\prime}$ with $\mathcal{E}_{0, k}(\bar{D}) \subset B_{k}^{\prime} \subset$ $\mathcal{E}_{0, k}(D)$ with the following properties:

(i) The spaces $B_{k}^{\prime}$ are closed under $T_{g}$.

(ii) If $k \geq 0$ and $f \in B_{k+1}+B_{k+1}^{\prime}$ and $\bar{\partial} f=0$, then there is a solution $u \in B_{k}^{\prime}$ to $\bar{\partial} u=f$.

(iii) If $f \in B_{0}+B_{0}^{\prime}$ and $\bar{\partial} f=0$, then $f \in X$.

To be precise, in [10] spaces $B_{k}^{\prime}$ are defined such that (i) and (ii) hold for $k \geq 1$ (for $k \geq 2$ actually $B_{k}^{\prime}=B_{k}$ ). Moreover, $B_{0}^{\prime}$ is chosen as $L^{p}(\partial D$ ) and the exact statement of (ii) for $k=0$ is: If $f \in B_{1}+B_{1}^{\prime}$ then there is a solution $u \in B_{0}^{\prime}$ to $\bar{\partial}_{b} u=f$; for this use of the symbol $\bar{\partial}_{b}$, see [10]. Moreover, it turns out that there is a trace mapping $\tau: B_{0} \rightarrow \tau B_{0} \subset L^{p}(\partial D)$, thus the elements in $B_{0}$ have boundary values in $L^{p}(\partial D)$, and the precise statement of (iii) is: If $f \in \tau B_{0}+B_{0}^{\prime}$ and $\bar{\partial}_{b} f=0$, then $f \in \tau H^{p} \simeq H^{p}$.

In the proof below, for simplicity, we assume that we actually have access to spaces satisfying (i) to (iii), and leave it to the reader to fill in the small formal modifications that are needed; see also [10].

Lemma 3.2. If $u \in \mathcal{L}^{-r}\left(\operatorname{Tot} B_{k}^{l}(D)\right)$ and

$$
(\delta-\bar{\partial}) u=f \in \Lambda^{r-1} X,
$$

then there is $v \in \mathcal{L}^{-r-1}\left(\operatorname{Tot}\left(B_{k}^{\prime}\right)^{l}(D)\right)$ such that

$$
u+(\delta-\bar{\partial}) v=h \in \Lambda^{r} X .
$$

Proof. In fact, if $u=\sum u_{r+k, k}$ with $u_{r+k, k} \in B_{k}^{r+k}$, then $\bar{\partial} u_{r+n, n}$ $=0$ so we can solve $\bar{\partial} v_{r+n, n-1}=u_{r+n, n}$ in $\left(B_{n-1}^{\prime}\right)^{r+n}$. Then $\delta v_{r+n, n-1} \in$ $\left(B_{n-1}^{\prime}\right)^{r+n-1}$ and $\bar{\partial}\left(u_{r+n-1, n-1}+\delta v_{r+n, n-1}\right)=0$, so we can inductively find $v_{r+k+1, k} \in\left(B_{k}^{\prime}\right)^{r+k+1}$ such that $\bar{\partial} v_{r+k, k-1}=u_{r+k, k}+\delta v_{r+k+1, k}$. Finally, $h=u_{r, 0}+\delta v_{r+1,0} \in \Lambda^{r} X$.

We can now conclude the proof of Theorem 2.1.

Proof of Theorem 2.1. We start with the injectivity. In view of (3.4) it is enough to show that the leftmost mapping actually is an isomorphism. Take $[f] \in H_{r}\left(T_{g}, X\right)$ and assume that it is 0 in $H^{-r}$ (Tot $\left.B_{k}^{l}(D)\right)$. Thus there is a $u \in \mathcal{L}^{-r-1}\left(\operatorname{Tot} B_{k}^{l}(D)\right)$ such that $(\delta-\bar{\partial}) u=f$. But then, by Lemma 3.2, there is $v \in \mathcal{L}^{-r-2}\left(\operatorname{Tot}\left(B_{k}^{\prime}\right)^{l}\right)$ such that

$$
u+(\delta-\bar{\partial}) v=h \in \Lambda^{r+1} X .
$$

Thus

$$
\delta h=(\delta-\bar{\partial}) h=(\delta-\bar{\partial}) u=f
$$

so that $[f]=0$ in $H_{r}\left(T_{g}, X\right)$. Thus $(2.2)$ is injective. 
For the surjectivity, take $[u] \in H^{-r}\left(\operatorname{Tot} B_{k}^{l}\right)$; then $($ since $(\delta-\bar{\partial}) u=0$ ) Lemma 3.2 gives a $v \in \mathcal{L}^{-r-1}\left(\operatorname{Tot}\left(B_{k}^{\prime}\right)^{l}\right)$ such that

$$
u+(\delta-\bar{\partial}) v=h \in \Lambda^{r} X
$$

then

$$
\delta h=(\delta-\bar{\partial}) h=(\delta-\bar{\partial}) u=0,
$$

so $h$ really defines a class $[h]$ in $H_{r}\left(T_{g}, X\right)$. Now,

$$
u-h=-(\delta-\bar{\partial}) v
$$

in $V$, i.e., $[u]$ and $[h]$ coincide as elements in $H^{-r}\left(\operatorname{Tot} \mathcal{E}_{0, k}^{l}(V)\right)$, and by (3.4) it follows that they actually coincide in $H^{-r}$ (Tot $\left.B_{k}^{l}(D)\right)$, and thus $[u]$ is in the image of (2.2). This concludes the proof.

For the reader who wants a more concrete argument for the isomorphism (2.2), let us dissect the argument; we restrict to the injectivity part. So let us again start with $[f] \in H_{r}\left(T_{g}, X\right)$ and assume that its image in $H_{r}\left(T_{g}, \mathcal{O}(V)\right)$ vanishes. Thus there is a holomorphic solution in $V$ to $\delta v=f$. With the notation from the proof of Lemma 3.1 we get the form

$$
w=\chi v+(1-\chi) \alpha \wedge f-\bar{\partial} \chi \wedge \alpha \wedge v \in \mathcal{L}^{-r-1}\left(\operatorname{Tot} B_{k}^{l}(D)\right)
$$

such that $(\delta-\bar{\partial}) w=f$. It remains to analyze what happens in the proof of Lemma 3.2. If $K$ is a linear homotopy operator for $\bar{\partial}$ in $D$ that admits the stated estimates, then the function $h \in \Lambda^{r+1} X$ such that $\delta h=f$ is obtained as

$$
h=\sum_{j=0}^{n}(\delta K)^{j} w_{j},
$$

where $w_{j}$ is the component of $w$ which is in $B_{j}^{-r-1+j}$. The surjectivity part can be explained in a very similar way.

One can also for simplicity carry out the whole procedure in the smaller domains $D_{\varepsilon}=\{\varrho<-\varepsilon\}$, so that $g$ is holomorphic in a neighborhood, and everything is smooth (or at least continuous) up to the boundary. Noticing that we have uniform $B_{k}$ estimates at each step, we end up with $h_{\varepsilon}$ with uniform $H^{p}\left(D_{\varepsilon}\right)$ estimates such that $\delta_{g} h_{\varepsilon}=f$ in $D_{\varepsilon}$. The conclusion then follows by a normal family argument.

\section{References}

[1] M. Andersson, The $H^{2}$ corona theorem, preprint, Göteborg, 1990.

[2] - On the $H^{p}$ corona problem, Bull. Sci. Math. 118 (1994), 287-306.

[3] M. Andersson and H. Carlsson, Estimates of solutions of the $H^{p}$ and BMOA corona problem, Math. Ann. 316 (2000), 83-102.

[4] - - - $\mathcal{Q}_{p}$ spaces in strictly pseudoconvex domains, J. Anal. Math. 84 (2001), 335-359. 
[5] J. Eschmeier and M. Putinar, Spectra of analytic Toeplitz tuples on Bergman spaces, Acta Sci. Math. (Szeged) 57 (1993), 85-101.

[6] —, - Spectral Decomposition and Analytic Sheaves, Oxford Sci. Publ., Oxford, 1996.

[7] J. M. Ortega and J. Fàbrega, Corona type decomposition in some Besov spaces, Math. Scand. 78 (1996), 93-111.

[8] - - - Pointwise multipliers and decomposition theorems in analytic Besov spaces, Math. Z. 235 (2000), 53-81.

[9] M. Putinar, On joint spectra of pairs of analytic Toeplitz operators, Studia Math. 115 (1995), 129-134.

[10] S. Sandberg, Property $(\beta)_{\mathcal{E}}$ for Toeplitz operators with $H^{\infty}$-symbol, J. Operator Theory, to appear.

[11] J. L. Taylor, A joint spectrum for several commuting operators, J. Funct. Anal. 6 (1970), 172-191.

[12] R. Wolff, Spectra of analytic Toeplitz tuples on Hardy spaces, Bull. London Math. Soc. 29 (1997), 65-72.

[13] J. Xiao, The $\mathcal{Q}_{p}$ corona problem, Pacific J. Math. 194 (2000), 491-509.

Department of Mathematics

Chalmers University of Technology

and Göteborg University

S-412 96 Göteborg, Sweden

E-mail:matsa@math.chalmers.se

sebsand@math.chalmers.se

Received September 21, 2001

Revised version September 3, 2002 